\title{
Análise Térmica da Poliacrilonitrila Plastificada com Glicerol em Extrusora
}

\author{
Carlos A. R. Brito Júnior, Robson R. Fleming \\ Pós-graduação em Engenharia Aeronáutica e Mecânica, ITA \\ Luiz C. Pardini \\ DCTA/IAE, ITA
}

Nilton P. Alves

Quimlab Científica Ltda

Resumo: Neste trabalho são apresentados resultados de análise térmica para poliacrilonitrila (PAN) plastificada com glicerina. Foram observados os efeitos da glicerina de alta pureza (glicerol) e demais aditivos à base de glicóis na fusão e degradação térmica da PAN pela técnica de DSC sob alta taxa de aquecimento $\left(90^{\circ} \mathrm{C} / \mathrm{min}\right)$ e atmosfera inerte. Foi constatado que o glicerol reduziu a temperatura de fusão da PAN de $290{ }^{\circ} \mathrm{C}$ para $217^{\circ} \mathrm{C}$. Em uma etapa preliminar foi empregado o método de Kissinger para verificar o efeito da composição química da PAN sob sua degradação térmica. A energia de ativação aparente para o copolímero de PAN foi calculada em 149 kJ.mol ${ }^{-1}$. Sugeriu-se que a presença do comonômero acetato de vinila (AV) na composição química da PAN proporcionou menor entalpia de degradação (353 J.g $\mathrm{g}^{-1}$ ) em comparação com outros comonômeros constituintes de uma PAN precursora de fibras de carbono (988 J.g ${ }^{-1}$ ).

Palavras-chave: Poliacrilonitrila, glicerina, análise térmica, método de Kissinger.

\section{Thermal Analysis of Extruded Polyacrylonitrile Plasticized by Glycerol}

\begin{abstract}
This paper presents results from thermal analysis of polyacrylonitrile (PAN) plasticized with glycerin, where effects from the high purity glycerin (glycerol) and other additives to glycol were studied. Using differential scanning calorimetry (DSC) at high heating rate $\left(90^{\circ} \mathrm{C} / \mathrm{min}\right.$ ) and inert atmosphere, we observed a decrease in the melting temperature of PAN (from $290{ }^{\circ} \mathrm{C}$ to $217^{\circ} \mathrm{C}$ ) owing to the presence of glycerol. The Kissinger's method was used to investigate the kinetics of thermal degradation for acrylonitrile (AN) with vinyl acetate (AV). The apparent activation energy for the copolymer AN/AV was calculated as $149 \mathrm{~kJ}^{\mathrm{mol}}{ }^{-1}$. The AV comonomer resulted in a

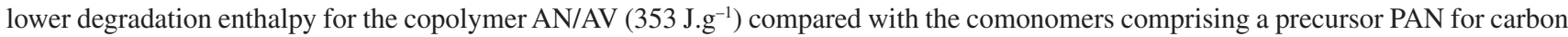

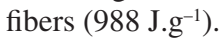

Keywords: Polyacrylonitrile, glycerin, thermal analysis, Kissinger's method.

\section{Introdução}

A poliacrilonitrila (PAN) é um copolímero sintético, sintetizado preferencialmente via polimerização radicalar a partir do monômero acrilonitrila $\mathrm{AN}\left(\mathrm{C}_{3} \mathrm{H}_{3} \mathrm{~N}\right)$. A inserção de comonômeros dando origem a copolímeros de PAN podem resultar materiais com novas propriedades, permitindo assim a modificação das propriedades da PAN tanto pelo tipo de comonômero como pela sua quantidade em relação ao monômero AN. Comonômeros ácidos (ácido itacônico (IA), por exemplo) aumentam a propriedade hidrofílica das fibras de PAN e reduzem a temperatura de degradação ${ }^{[1]}$. A presença de um comonômero neutro como o acetato de vinila (AV) no copolímero de PAN (Figura 1) pode aumentar a solubilidade da PAN em vários solventes orgânicos empregados em processos de fiação da solução $0^{[2,3]}$.

Sob aquecimento gradual, antes de atingir a temperatura de fusão (entre 320 a $330^{\circ} \mathrm{C}$ ), ocorre a ciclização dos grupos nitrilas adjacentes na cadeia molecular e subseqüentemente a degradação do polímero $\mathrm{PAN}^{[4,5]}$. O mecanismo de degradação da PAN é complexo e há discordâncias na literatura quanto a ordem das principais reações envolvidas na degradação térmica (ciclização, desidrogenação e oxidação $)^{[6-10]}$. Fitzer ${ }^{[9]}$, por exemplo, considera que a desidrogenação precede a ciclização na PAN sob atmosfera oxidante. Enquanto que Xue ${ }^{[10]}$ atribui a ciclização dos grupos nitrila da PAN como a primeira reação ocorrida durante a estabilização térmica. Denomina-se de estabilização térmica (estabilização oxidativa ou pré-oxidação) ao primeiro estágio do tratamento térmico para converter as fibras de PAN em fibras de carbono e corresponde, portanto, ao controle da degradação térmica. Essa etapa ocorre geralmente entre 180 a $300{ }^{\circ} \mathrm{C}$ em atmosfera que contenha oxigênio ${ }^{[8,11]}$. Coleman et al. ${ }^{[12-15]}$ propuseram que a degradação térmica da PAN sob vácuo inicia-se próximo a $160{ }^{\circ} \mathrm{C}$. Masson ${ }^{[16]}$ atribui que a degradação térmica da PAN é consequiência de sua natureza altamente polar.

A característica intrínseca de degradação da PAN quando próxima da sua temperatura de fusão impossibilita seu emprego em processos de transformação por fusão (extrusão, injeção, termo-conformação, entre outros). Somente eram apropriados para PAN os métodos de transformação (fiação úmida, fiação a seco e eletrofiação) que utilizassem solventes orgânicos como, por exemplo, a dimetilformamida (DMF).

Desde o início da produção das fibras de PAN (em 1950 pela DuPont) que diversos métodos para sua fusão sem uso de solventes orgânicos foram propostos em trabalhos e patentes ${ }^{[17-32]}$. Em muitos desses estudos foi proposto um processo para fiar a PAN no estado fundido, empregando um meio aquoso sob condições de alta temperatura e pressão ${ }^{[17-23,27,28]}$. Cox ${ }^{[17]}$ foi o primeiro a verificar que a temperatura de fusão do homopolímero de PAN reduzia de 320 a

Autor para correspondência: Carlos A. R. Brito Júnior, Pós-graduação em Engenharia Aeronáutica e Mecânica, Instituto Tecnológico de Aeronáutica - ITA, Praça Marechal Eduardo Gomes, 50, Vila das Acácias, CEP 12228-900, São José dos Campos, SP, Brasil, e-mail: riosbritojunior@yahoo.com.br 
$200{ }^{\circ} \mathrm{C}$ com $0,2 \%$ de concentração em massa de água. Entretanto, a homogeneidade da fiação foi extremamente comprometida em consequiência da liberação de vapor na fieira e formação de espuma. O processo de Cox foi modificado por Porosoff ${ }^{[19]}$ na tentativa aperfeiçoar o método de extrusão da PAN plastificada com água. Foi utilizado um sistema de extrusão com uma zona de solidificação das fibras submetida a alta pressão. Esse processo permitiu a fiação da PAN em meio livre de solvente orgânico com bom estiramento das fibras ${ }^{[19]}$. Contudo, Grove et al. ${ }^{[23]}$ observaram que as fibras obtidas pelo método de Porosoff apresentavam numerosos defeitos internos e de superfície (vazios com diâmetro de até $4 \mu \mathrm{m}$ foram observados próximo ao núcleo das fibras). Daumit et al. da $\operatorname{BASF}^{[24,25]}$ patentearam um método para fusão da PAN que demonstrou eficiência na obtenção de filamentos com uma estrutura fibrilar adequada para conversão de fibras de carbono. O processo da BASF consistia no emprego de uma mistura de água, acetonitrila (que posteriormente foi substituído por nitrometano e nitroetano) e um monohidróxi-álcool. Essa mistura atuaria na plastificação da PAN reduzindo seu ponto de fusão entre 160 a $185^{\circ} \mathrm{C}$. O processo requer uma câmara pressurizada a até $345 \mathrm{kPa}$. Essa câmara é usada para controlar o lançamento do plastificante e reduzir a formação de vazios durante a formação inicial das fibras. A técnica é eficiente somente para copolímeros de PAN com concentração em massa de AN entre 85 a $90 \%$. Esse método requer até $47 \%$ do plastificante que apresenta toxidade e, portanto, requer cuidado no manuseio. Necessariamente, uma estação para recuperação do plastificante deve ser instalada. Consequentemente, as fibras de PAN apresentam um preço final elevado. Portanto, devido às razões mencionadas anteriormente, as fibras de PAN obtidas pelo processo da BASF nunca foram comercializadas.

Outros trabalhos procuravam obter copolímeros de PAN adequados a um processo parcial de fusão e fiação com redução da massa molar por agentes à base de mercaptana ${ }^{[29,30]}$. Estes copolímeros de PAN altamente termoplásticos foram desenvolvidos pela Companhia Bristish Petroleum (BP) sob a marca Amlon ${ }^{\circledR}$. O Amlon ${ }^{\circledR}$ pode ser extrudado e fiado diretamente em uma extrusora comum. Contudo, é necessário taxas de alimentação extremamente baixas, comprometendo significantemente a eficiência da produção.

Atualmente, algumas pesquisas buscam alternativas para substituição de solventes para PAN empregando líquidos iônicos com baixa volatilidade como, por exemplo, o brometo de 1-butil-3-metilimidazólio e o cloreto de 1-butil-3-metilimidazólio, capazes de solubilizar a PAN e garantir um comportamento reológico adequado para um processo de fiação ${ }^{[31,32]}$. Os líquidos iônicos não apresentam alta toxidade se comparados com os solventes orgânicos sintéticos ${ }^{[33]}$. Entretanto, o alto custo dos líquidos iônicos inviabiliza seu emprego na indústria de fibras de PAN.

Em 2006, Alves ${ }^{[34]}$ observou que o álcool 1,2,3 propanotriol (glicerol) poderia atuar como plastificante da poliacrilonitrila permitindo sua fusão em um processo de extrusão. Por meio deste método é possível produzir grãos de PAN contendo a formulação na concentração adequada para uso em processos de transformação termoplástica como, por exemplo, moldagem por

$$
\begin{aligned}
& {\left[\mathrm{CH}_{2}-\mathrm{CH}\right]_{\mathrm{n}}\left[\mathrm{CH}_{2}-\mathrm{CH}_{\mathrm{m}}\right.} \\
& \mathrm{C} \equiv \mathrm{N} \quad \text { O } \\
& \mathrm{C}=\mathrm{O} \\
& \text { I } \\
& \mathrm{CH}_{3}
\end{aligned}
$$

Figura 1. Molécula de poliacrilonitrila- $c o$-acetato de vinila. injeção, extrusão de perfis semi-acabados, moldagem por sopro e fiação. Esta técnica não utiliza qualquer solvente orgânico sintético, possibilitando uma redução de custo para produção de fibras de PAN em até $1 / 3$ do custo atual. Sob o aspecto humano, este processo contribui significantemente para a saúde dos operadores que deixam de manusear solventes altamente tóxicos. A substituição destes solventes por aditivos a base de glicóis evita os riscos de contaminação ao meio-ambiente. Além disso, o uso do glicerol como plastificante da PAN em escala industrial torna-se mais uma alternativa como rota de consumo para a glicerina produzida como subproduto pela indústria de biodiesel.

O objetivo principal do presente trabalho é verificar o comportamento referente a degradação térmica da PAN sob a presença da glicerina pura (glicerol) e outros aditivos que atuam como plastificante pela técnica de calorimetria exploratória diferencial (DSC). As informações compiladas por este método são essencialmente importantes para o desenvolvimento do processo de fiação por fusão em extrusora para PAN. Inicialmente, em outro caso, uma análise térmica e cinética deve ser realizada para avaliar o efeito da composição química da PAN usada neste trabalho. As informações referentes aos dados cinéticos calculados a partir do método de Kissinger para PAN podem auxiliar na escolha de uma composição química da PAN adequada ao novo método de fiação por fusão.

\section{Experimental}

\section{Materiais}

Foi empregado o copolímero de poliacrilonitrila com acetato de vinila (PAN-co-AV) na forma de pó com composição mássica de AV de 6\% e massa molar média $\mathrm{M}_{\mathrm{n}}$ de 138.000 g. $\mathrm{mol}^{-1}$. Este copolímero de PAN foi fornecido pela empresa Radicifibras (SP/Brasil) e apresenta um custo menor se comparado com a PAN precursora para fibras de carbono (FC), conforme Tabela 1.

Para essa análise térmica foram empregadas como amostras comparativas:

- Homopolímero de PAN $\left(\mathrm{M}_{\mathrm{n}}=270.000 \mathrm{~g} \cdot \mathrm{mol}^{-1}\right)$ fornecido pela empresa Radicifibras (SP/Brasil); e

- Fibras de PAN precursoras de fibras de carbono fornecidas pela companhia inglesa Courtaulds sob o nome Courtelle. Essas fibras foram obtidas pelo processo de fiação a úmida. Apresenta massa molar $\mathrm{M}_{\mathrm{n}}$ de 100.000 g.mol ${ }^{-1}$ e composição mássica de $93 \%$ de AN, $5,3 \%$ de MA e $1,7 \%$ de IA $^{[35]}$.

Como plastificante da PAN foi usado o glicerol grau analítico (99,5\% de pureza) com $\mathrm{M}_{\mathrm{n}}$ de $92 \mathrm{~g} \cdot \mathrm{mol}^{-1}$, ponto de fusão a $18{ }^{\circ} \mathrm{C}$ e ponto de ebulição aproximadamente a $290{ }^{\circ} \mathrm{C}^{[36]}$. Fornecido pela empresa Vetec Química Fina (RJ/Brasil).

Tabela 1. Alguns comônomeros empregados na polimerização com AN.

\begin{tabular}{ccc}
\hline Comonômero & Empregabilidade & $\begin{array}{c}\text { Distribuidora } \\
\text { (*Preço/kg) }\end{array}$ \\
\hline $\begin{array}{c}\text { Metacrilato de metila } \\
\text { (MMA) }\end{array}$ & $\begin{array}{c}\text { Precursor de FC; } \\
\text { Têxtil }\end{array}$ & Lucite (US\$ 5,00) \\
Acrilato de metila & Precursor de FC & Arkema (US\$ 4,40) \\
(MA) & Têxtil; Terpolímero & Brazmo (US\$ 2,14) \\
Estireno (ES) & ABS & Vetta Química (US\$ \\
Ácido itacônico (IA) & Precursor de FC & $2,60)$ \\
Acetato de vinila (AV) & Têxtil & Dow (US\$ 2,80) \\
\hline
\end{tabular}

*Cotação interna realizada pela empresa Quimlab (SP/Brasil) em 2009. 


\section{Métodos}

\section{Preparo dos grãos de PAN plastificada com glicerol}

O copolímero de PAN foi misturado, em maior proporção, com o plastificante glicerol (em até $30 \%$ da massa total) e outros aditivos a que alimentou uma extrusora laboratorial tipo monorosca com capacidade de produção de $2 \mathrm{~kg} / \mathrm{h}$. A rosca extrusora empregada apresentou um perfil com quatro estágios ou zonas (de alimentação, de compressão, de degasagem e de dosagem) e uma relação L/D (comprimento/diâmetro) de 32. O projeto da extrusora foi executado pela fabricante AX Plásticos (SP/Brasil). A descrição da proporção mássica e dos demais detalhes quanto ao processo de extrusão da PAN são de propriedade intelectual da empresa Quimlab ${ }^{[34,37]}$. PAN com glicerol foi fundida durante a extrusão com a temperatura do processo entre 200 a $220{ }^{\circ} \mathrm{C}$. Para conformar a PAN fundida em monofilamento, foi acoplado ao final da extrusora uma matriz apropriada com um canal de $45 \mathrm{~mm}$ de comprimento, diâmetro na entrada de $12 \mathrm{~mm}$ e diâmetro de saída de $6 \mathrm{~mm}$. O monofilamento de PAN foi puxado e alongado por meio de dois rolos de poliuretano que reduziram a sua seção transversal de 6 para $3 \mathrm{~mm}$ de diâmetro. Em seguida este monofilamento foi seccionado ao atravessar um equipamento com facas rotativas por onde foram obtidos grãos de PAN plastificada. Estes grãos foram coletados e armazenados.

\section{Análise térmica qualitativa da PAN por DSC}

$\mathrm{O}$ equipamento utilizado para investigação das transições térmicas da PAN foi o modelo DSC-60 da fabricante Shimadzu localizado no laboratório da empresa Quimlab (SP/Brasil). A calibração deste equipamento foi realizada com padrões de zinco e índio com temperaturas de fusão de $419,50{ }^{\circ} \mathrm{C}$ e $156,60{ }^{\circ} \mathrm{C}$, respectivamente.

Todas as amostras foram colocadas em panelas de alumínio e pesadas em balança analítica com as massas variando, para cada amostra, entre 2,0 a 2,4 mg. As panelas foram seladas em prensa hidráulica. O fluxo de gás argônio usado em todas as análises por DSC foi ajustado para $25 \mathrm{~mL} / \mathrm{min}$.

Para verificar a temperatura de fusão sob análise por DSC, a taxa de aquecimento de $90{ }^{\circ} \mathrm{C} / \mathrm{min}$ foi empregada para as amostras de PAN plastificada, homopolímero de PAN e copolímero de 20 e $25^{\circ} \mathrm{C} / \mathrm{min}$ forneceram valores de entalpia e temperatura de degradação $\left(\mathrm{T}_{\mathrm{d}}\right)$ para as amostras de copolímero de PAN-co-AV e fibras de PAN precursoras. A aquisição dos dados para todas as amostras foi programada para iniciar a $30^{\circ} \mathrm{C}$ e finalizar a $450{ }^{\circ} \mathrm{C}$.

A norma ASTM E 698 ${ }^{[38]}$ recomenda para o cálculo dos parâmetros cinéticos os métodos de Ozawa e Kissinger. Neste trabalho arbitrou-se pelo método de Kissinger ${ }^{[39]}$ assim como nos trabalhos de $\mathrm{Hou}^{[40]}$ e Ouyang ${ }^{[41]}$ que empregaram somente 4 taxas de aquecimento com eventual sucesso na determinação dos parâmetros cinéticos para copolímeros de PAN. Portanto, foi usado na determinação da energia de ativação aparente $\Delta E_{a}$ para o copolímero de PAN a seguinte equação ${ }^{[38-41]}$ :

$$
-\frac{\Delta E_{a}}{R}=\frac{d \ln \left(\frac{\Phi}{T_{d}^{2}}\right)}{d\left(\frac{1}{T_{d}}\right)}
$$

Onde $\Phi$ é a taxa de elevação da temperatura (taxa de aquecimento), $T_{d}$ é a temperatura absoluta do pico exotérmico para cada taxa de aquecimento (referente a temperatura de degradação da PAN) e $R$ é a constante dos gases $\left(8,31447 \mathrm{~J} \cdot \mathrm{mol}^{-1} \cdot \mathrm{K}^{-1}\right)$. A energia base de glicóis ${ }^{[34]}$. Assim, foi formada uma "massa de pré-extrusão" PAN-co-AV. Enquanto que as taxas de aquecimento de 5, 10,

de ativação aparente da degradação para o polímero PAN pode ser determinada pelo declive de um gráfico linear de $\ln \left(\Phi \cdot T_{d}{ }^{-2}\right)$ em função de $10^{3} \cdot T_{d}^{-1}$. Ou seja, o coeficiente $\operatorname{angular}(m)$ da equação da reta $(y=m x+b)$ pode ser relacionado como:

$$
m=-\frac{E_{a}}{R}
$$

O fator pré-exponencial de Arrhenius ( $k$ ) também pode ser associado à equação da reta pela relação com o coeficiente linear $\mathrm{b}=\ln \left(\mathrm{AR} / \mathrm{T}_{\mathrm{d}}\right)$ ou pela Equação 3 (segundo a norma ASTM E 698-79 $\left.{ }^{[38]}\right)$ :

$$
k=\frac{\Phi E_{a} e^{E_{a} / R T_{d}}}{R T_{d}^{2}} \cdot\left(s^{-1}\right)
$$

\section{Resultados e Discussões}

\section{Caracterização do copolímero de PAN}

Todas as curvas DSC fornecidas para taxas de aquecimento de 5 a $25^{\circ} \mathrm{C} / \mathrm{min}$. exibiram, cada uma, um único pico exotérmico, correspondente à degradação térmica da PAN.

A energia de ativação aparente de degradação térmica para as amostras de PAN foram calculados a partir dos parâmetros descritos no gráfico na Figura 2. O valor da $\mathrm{E}_{\mathrm{a}}$ resultou próximo a $149 \mathrm{~kJ} \cdot \mathrm{mol}^{-1}$ para o copolímero de PAN-co-AV e $115 \mathrm{~kJ} \mathrm{~mol}^{-1}$ para amostra de fibras de PAN precursora fornecida pela Courtaulds.

A Tabela 2 resumiu uma comparação entre os valores obtidos por DSC e os parâmetros cinéticos calculados para as amostras de PAN. A temperatura absoluta do pico de degradação $T_{d}$ e a variação para entalpia de degradação $\Delta \mathrm{H}$ foram obtidos para taxa de aquecimento de $10{ }^{\circ} \mathrm{C} / \mathrm{min}$ para todas as amostras.

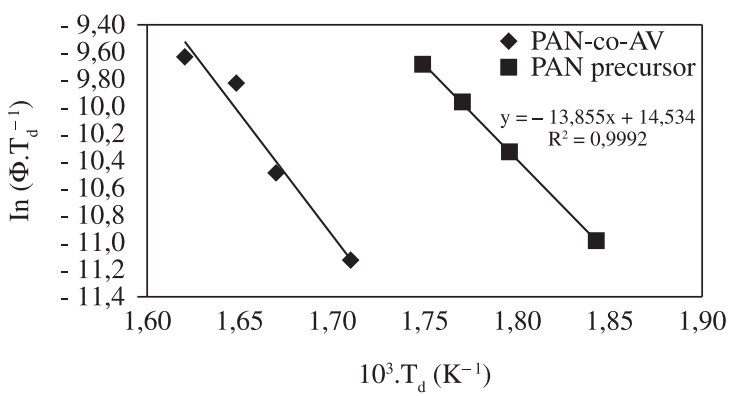

Figura 2. Gráfico referente ao método de Kissenger para determinação da energia de ativação da degradação térmica do copolímero PAN-co-AV e da PAN precursora de fibra de carbono.

Tabela 2. Valores para analise térmica da PAN em DSC a taxa de $10^{\circ} \mathrm{C} / \mathrm{min}$ em atmosfera de $\mathrm{Ar}$ (os precursores 2 e 3 são referências de artigo e foram analisados em atmosfera de $\mathrm{N}_{2}$ ).

\begin{tabular}{cccccc}
\hline Amostra & $\begin{array}{c}\mathbf{T}_{\text {inicial }} \\
\left({ }^{(} \mathbf{C}\right)\end{array}$ & $\begin{array}{c}\mathbf{T}_{\mathbf{d}} \\
\left({ }^{\circ} \mathbf{C}\right)\end{array}$ & $\begin{array}{c}\mathbf{E}_{\mathrm{a}} \\
\left(\mathbf{k J} \cdot \mathbf{m o l}^{-1}\right)\end{array}$ & $k\left(\mathbf{s}^{-1}\right)$ & $\begin{array}{c}\Delta \mathbf{H} \\
\left(\mathbf{J J g}^{-1}\right)\end{array}$ \\
\hline $\begin{array}{c}\text { PAN-co-AV } \\
(6 \% \mathrm{AV})\end{array}$ & 298 & 326 & 149 & $5,3 \times 10^{12}$ & 353 \\
$\begin{array}{c}\text { Precursor 1/ } \\
\text { Courtaulds }\end{array}$ & 234 & 284 & 115 & $2,7 \times 10^{10}$ & 988 \\
$\begin{array}{c}\text { "Precursor 2/ } \\
\text { Mitsubishi }\end{array}$ & --- & 259 & 127 & $1,5 \times 10^{12}$ & 950 \\
"Precursor 3/ & --- & 282 & 140 & $8,1 \times 10^{12}$ & 939 \\
Asahi Kasei & & & & & \\
\hline *Referência: Zhang et al. ${ }^{[42]}$ & & &
\end{tabular}


Conforme a Tabela 2, a PAN copolimerizada com AV apresentou maior energia de ativação aparente de degradação. Conseqüentemente, pode ser necessário fornecer maior quantidade de calor para iniciar a cisão do grupamento $\mathrm{C} \equiv \mathrm{N}$ na cadeia molecular da PAN. Portanto, a temperatura inicial para conversão das fibras de PAN-co-AV em fibras de carbono deve ser maior que $290{ }^{\circ} \mathrm{C}$. $O$ fator pré-exponencial $k$ indicou que a reação de degradação na PAN-co-AV ocorre com menor velocidade que os precursores 1 e 2. A reação de degradação da PAN-co-AV ocorre com menor variação de entalpia que os demais precursores. Portanto, é pressuposto que há menor liberação de calor durante a formação dos anéis cíclicos (ciclização) na cadeia da PAN devido à presença do AV.

Observando a temperatura inicial de degradação $\left(\mathrm{T}_{\text {inicial }}\right)$ pode-se deduzir que o comonômero AV retarda o início das reações relacionadas a degradação da PAN mais eficientemente que os comonômeros MA e IA. Isso é importante para a extrusão da PAN, uma vez que a degradação térmica é um evento indesejável neste processo.

Um trabalho apresentado por Han et al. ${ }^{[43]}$ mostra que a concentração de $15 \%$ em massa de AV no copolímero PAN fornece uma $\mathrm{T}_{\mathrm{d}}$ de aproximadamente $292{ }^{\circ} \mathrm{C}$ a taxa de aquecimento de $10{ }^{\circ} \mathrm{C} /$ min e atmosfera de $\mathrm{N}_{2}$. Confrontando este valor $\left(\mathrm{T}_{\mathrm{d}}=292{ }^{\circ} \mathrm{C}\right)$ do copolímero PAN-co-AV com o valor encontrado no presente trabalho $\left(\mathrm{T}_{\mathrm{d}}=326^{\circ} \mathrm{C}\right)$ nota-se que a temperatura do pico exotérmico reduz com o aumento da concentração de $\mathrm{AV}$.

\section{Caracterização da PAN plastificada com glicerol em processo de extrusão}

Conforme literatura ${ }^{[16]}$, entre as taxas de aquecimento de 40 a $160{ }^{\circ} \mathrm{C} / \mathrm{min}$. pode-se observar o ponto de fusão para o homopolímero PAN. Foi arbitrado uma taxa de aquecimento de $90{ }^{\circ} \mathrm{C} /$ min para verificar no gráfico de DSC a fusão no copolímero de PAN. A Figura 3 apresentou três curvas fornecidas por DSC em atmosfera inerte de argônio referentes ao homopolímero de PAN, ao copolímero PAN-co-AV (6\% AV) e a este mesmo copolímero plastificado com glicerol.

Não foi possível identificar na Figura 3 um pico referente à fusão do homopolímero de PAN, somente o pico referente à degradação em $366{ }^{\circ} \mathrm{C}$, sob a taxa de aquecimento utilizada. Provavelmente, devido ao início da reação de degradação que ocorre muito próximo a temperatura de fusão, como citado em literatura ${ }^{[4,5]}$. Entretanto, foi observado um pico endotérmico na curva DSC para o copolímero de PAN em $291{ }^{\circ} \mathrm{C}$ e outro em $217{ }^{\circ} \mathrm{C}$ para o copolímero de PAN plastificado com glicerol. Atribuiu-se a estes picos a transição térmica relacionada a fusão. Supostamente, a presença do glicerol

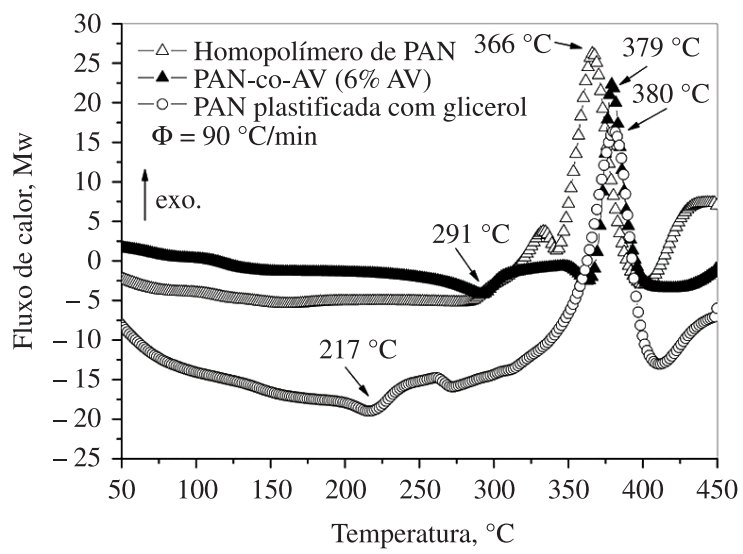

Figura 3. Curvas DSC para amostras de PAN sob taxa de aquecimento de $90{ }^{\circ} \mathrm{C} / \mathrm{min}$ em atmosfera inerte (gás argônio). conduziu a uma menor temperatura de fusão da PAN. A análise na Figura 3 mostrou ainda que a presença do glicerol não alterou significativamente a $T_{d}$ da PAN. Isso ocasionou um tempo entre as transições térmicas (fusão-degradação) de 1 minuto e 45 segundos para PAN com glicerol e 1 minuto para PAN sem glicerol (intervalo correspondente a $\Phi=90^{\circ} \mathrm{C} / \mathrm{min}$ ).

Geralmente, análises por DSC não exibem resultados equivalentes para transições térmicas de um determinado material sob uma condição térmico dinâmica, como ocorre, por exemplo, nas análises por reometria, entre outras. Ou seja, a influência de outros parâmetros sob alguma propriedade térmica de um material, como por exemplo, a taxa de cisalhamento, pode ser considerada desprezível. Entretanto, no caso deste trabalho, a análise de DSC da PAN plastificada com glicerol revelou as propriedades térmicas adquiridas durante o processo de extrusão. Portanto, em processamento, o conhecimento das propriedades térmicas dos pellets da PAN é importante no processo de moldagem por injeção ou para um processo de re-extrusão (reciclagem), por exemplo.

\section{Conclusões}

O copolímero de PAN (AN/AV) necessitou de maior quantidade de calor $\left(149 \mathrm{~kJ} \cdot \mathrm{mol}^{-1}\right)$ para iniciar a reação de ciclização que o terpolímero AN/MA/IA (115 kJ.mol $\left.{ }^{-1}\right)$.

A presença do AV ocasionou uma menor liberação de energia

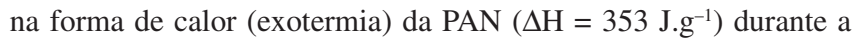
sua degradação térmica. Menor entalpia pode conduzir a um melhor controle no processo de estabilização oxidativa das fibras de PAN para obtenção de fibras de carbono.

A presença do glicerol proporcionou uma redução na temperatura de fusão da PAN. Essa condição pode proporcionar um maior tempo de permanência da PAN, sem degradação, em um processo de moldagem por injeção termoplástica ou re-extrusão.

\section{Agradecimentos}

A Quimlab e Radicifibras pelas amostras, ao Laboratório de Tecnologia do ITA, a FAPESP e CNPq pelo apoio financeiro.

\section{Referências Bibliográficas}

1. Bajaj, P.; Paliwal, D. K. \& Gupta, A. K. - J. Macromol. Sci. Chem.,

2. "Ullmann's Encyclopedia of Chemical Technology", 5th edition, VCH, Weinheim (1987).

3. Rajalingam, P. \& Radhakrishnan, G. - JMS-REV. Macromol. Chem. Phys., 283, p.301 (1991).

4. Clarke, A. J. \& Bailey, J. E. - Nature, 243, p.146 (1973). http://dx.doi. org/10.1038/243146a0

5. Hinrichsen, G.- J. Appl. Polym. Sci., 17, p.3305 (1973). http://dx.doi. org/10.1002/app.1973.070171106

6. Farsani, R. E.; Shokuhfar, A. \& Sedghi, A. - PWASET, 26, p.513 (2007).

7. Farsani, R. E. et al. - WASET, 50, p.430 (2009).

8. Mathur, R. B.; Bahl, O. P. \& Sivaram, P. - Curr. Sci., 62, p.662 (1992).

9. Fitzer, E. \& Muller, D. J. - Carbon, 13, p.63 (1975). http://dx.doi. org/10.1016/0008-6223(75)90259-6

10. Xue, T. J.; McKinney, M. A. \& Wilkie, C. A. - Polym. Degrad. Stab., 58, p.193 (1997). http://dx.doi.org/10.1016/S0141-3910(97)00048-7

11. Yu, M.; Wang, C.; Bai, Y.; Wang, Y.; Wang, Q. \& Liu, H. - Polym. Bull., 57, p.525 (2006). http://dx.doi.org/10.1007/s00289-006-0581-8

12. Coleman, M. M. \& Petcavich, R. J. - J. Polym. Sci.: Polym. Phys. Edn., 16, p. 821 (1978). http://dx.doi.org/10.1002/pol.1978.180160507 
13. Petcavich, R. J.; Painter, P. C. \& Coleman, M. M. - J. Polym. Sci.: Polym. Phys. Edn., 17, p.165 (1979). http://dx.doi.org/10.1002/ pol.1979.180170115

14. Coleman, M. M. \& Sivy, G. T. - Carbon, 19, p.123, 1981. http://dx.doi. org/10.1016/0008-6223(81)90118-4

15. Coleman, M. M.; Sivy, G. T.; Painter, P. C.; Snyder, R. W. \& Gordon III, B. - Carbon, 21, p.255 (1983). http://dx.doi.org/10.1016/00086223(83)90089-1

16. Masson, J. C. - "Acrylic Fiber Technology and Applications", Marcel Dekker, New York (1995).

17. Coxe, C. D. \& Wilmington, D. E. - "Preparation of shaped articles from acrylonitrile polymers", US Patent 2585444, 12 (1952).

18. Blickenstaff, R. A. - "Acrylonitrile polymer filaments", US Patent 3984601, n.294, 184 (1976).

19. Porosoff, H. - "Melt spinning acrylonitrile polymers fibers", US Patent 4163770 (1979).

20. DeMaria, F. \& Young, C. C. - "Process for melt spinning acrylonitrile polymer fiber using hot water as stretching aid", US Patent 4303607 (1981).

21. Pfeiffer, R. E. \& Peacher, S. E. - "Spinnerette plate having multiple capillaries per counterbore for melt spinning fusion melts of acrylonitrile polymer and water", US Patent 4318680 (1982).

22. Frushour, B. G. - Polym. Bull. 7, p.1 (1982).

23. Grove, D.; Desai, P.; Abhiraman, A. S. - Carbon, 26, p.403 (1988). http://dx.doi.org/10.1016/0008-6223(88)90233-3

24. Daumit, G. P. et al. - "Formation of melt-spun acrylic fibers which are particularly suited for thermal conversion to high strength carbon fibers", US Patent 4921656 (1990).

25. Daumit, G. P. et al. - "Melt-spun acrylic fibers which are particularly suited for thermal conversion to high strength carbon fibers", US Patent 4981751 (1991).

26. Atureliya, S. K. \& Bashir, Z. - Polymer, 34, p.5116 (1993). http:// dx.doi.org/10.1016/0032-3861(93)90256-A

27. Yoon, H. S. et al. - "Non-spun, short, acrylic polymer, fibers", US Patent 5434002, n.148, 629 (1995).

28. Yoon, H. S. et al. - "Unspun acrylic staple fibers", US Patent 5589264 , n.446, 287 (1996).
29. Smierciak, R. C.; Wardlow, J. E. \& Ball, L. E. - "A process for making a high nitrile multipolymer prepared from acrylonitrile and olefinically unsaturated monomers", WO Patent 1996/02/6968 (1996).

30. Smierciak, R. C.; Eddie, W. J. \& Ball, L. E. - "Process for making an acrylonitrile, methacrylonitrile and olefinically unsaturated monomers", US Patent 5602222, n.533, 230 (1997).

31. Liu, W.; Cheng, L.; Zhang, H.; Zhang, Y.; Wang, H. \& Yu, M. - Int. J. Mol. Sci., 8, p.180 (2007). http://dx.doi.org/10.3390/i8030180

32. Yang, T.; Yao, Y.; Lin, Y.; Wang, B.; Xiang, R.; Wu, Y. \& Wu, D. - Appl. Phys. A: Mater. Sci. Process., 98, p.517 (2009). http://dx.doi. org/10.1007/s00339-009-5483-9

33. Rogers, R. D. \& Sedden, K. R. - Science, 302, p.792 (2003). Pmid:14593156. http://dx.doi.org/10.1126/science.1090313

34. Alves, N. P. - W.O Patent 147224 (2007).

35. Wu, G. P. - Polym. Bull., 62, p.667 (2009). http://dx.doi.org/10.1007/ s00289-009-0039-x

36. Beatriz, A.; Araújo, Y. J. K. \& Lima, D. P. - Quim. Nova, 34, p.306 (2011). http://dx.doi.org/10.1590/S0100-40422011000200025

37. Alves, N. P. - U.S Patent 0024939 A1 (2011).

38. American Society for Testing and Materials - ASTM. ASTM E 698-79: Arrhenius kinetic constants for thermally unstable material. Philadelphia (1984).

39. Lee, J. S.; Hsu, C. K. \& Chang, C. L. - Thermochim. Acta, 173, p.392 (1973).

40. Hou, C.; Qu, R.; Qun, W.; Ying, L. \& Wang, C. - J. Appl. Polym. Sci., 98, p.1708 (2005). http://dx.doi.org/10.1002/app.22343

41. Ouyang, Q.; Cheng, L.; Wang, H. \& Li, K. - Polym. Degrad. Stab., 93, p.1415 (2008). http://dx.doi.org/10.1016/j. polymdegradstab.2008.05.021

42. Zhang, W. \& Liu, J. - J. Wuhan Univer. Technol. - Mat. Sci. Ed., 21, p.26 (2006).

43. Han, N.; Zhang, X.-X. \& Wang, X-C. - Iran. Polym. J., 19, p.243 (2010).

Enviado: 28/01/11

Reenviado: $27 / 09 / 11$ Aceito: 07/11/11 